\title{
Efficient Algorithms for Incremental Utility Mining
}

\author{
Jieh-Shan Yeh \\ Department of Computer Science \\ and Information Management \\ Providence University \\ Taichung 433, Taiwan \\ +886-4-26328001 \\ jsyeh@pu.edu.tw
}

\author{
Chih-Yang Chang \\ Department of Computer Science \\ and Information Management \\ Providence University \\ Taichung 433, Taiwan \\ +886-4-26328001 \\ g9471069@pu.edu.tw
}

\author{
Yao-Te Wang \\ Department of Computer Science \\ and Information Management \\ Providence University \\ Taichung 433, Taiwan \\ +886-4-26328001 \\ ytwang@pu.edu.tw
}

\begin{abstract}
Temporal data mining is the activity of finding interesting correlations or patterns in large temporal data sets. On the other hand, utility mining aims at identifying the itemsets with high utilities. In 2006, Tseng et al. introduced the temporal utility mining which is extended from both temporal association rule mining and utility mining. In this study, we investigated the incremental utility mining which can identify all high temporal utility itemsets in a specified time period on an incremental transaction database. Two efficient algorithms, Incremental Utility Mining (IUM) and Fast Incremental Utility Mining (FIUM), were proposed. The experimental results also showed that both algorithms are efficient.
\end{abstract}

\section{Categories and Subject Descriptors}

H.2.8 [Database Management]: Database Applications - data mining.

\section{General Terms}

Algorithms, Performance, Experimentation, Theory.

\section{Keywords}

Temporal data mining, incremental mining, utility mining.

\section{INTRODUCTION}

In traditional association rule mining model, the products of transactions are treated as items. Association rule mining identifies relationship among a set of items frequently purchased together. Apriori [1] is the most famous algorithm for finding association rules. Some researchers continued to improve it afterward, such as [2], [3], [4], [5], [6].

However, in many applications, mining temporal association patterns from the most recent data is also important. The concept of incremental mining was introduced by Cheung et al. [7] in

Permission to make digital or hard copies of all or part of this work for personal or classroom use is granted without fee provided that copies are not made or distributed for profit or commercial advantage and that copies bear this notice and the full citation on the first page. To copy otherwise, or republish, to post on servers or to redistribute to lists, requires prior specific permission and/or a fee.
1996. FUP Algorithm was proposed to solve such problem. Afterward, there were many researches on incremental sequential pattern mining aimed at the cases of incremental databases [8], [9], [10], [11], [12], [13].

Utility mining [14] is the research which discusses the quantity of items. Utility is defined as how useful or valuable an item is. Utility mining identifies high utility itemsets that own a large portion of the total utility in the transaction database. By combining the concept of temporal data mining and utility mining, Tseng et al. [15] introduced THUI-Mine algorithm for finding temporal high utility itemsets from data streams.

In this paper, we investigated the incremental utility mining which can identify all high temporal utility itemsets in a specified time period on an incremental transaction database. This study proposed two novel algorithms, Incremental Utility Mining (IUM) Algorithm and Fast Incremental Utility Mining (FIUM) Algorithm, which can discover all high temporal utility itemsets.

The rest of this paper is organized as follows. Section 2 overviews the related works. Section 3 describes the proposed algorithms. Performance studies are given in Section 4. Finally, we conclude in Section 5 with a summary of our work.

\section{RELATED WORKS}

\subsection{Utility Mining}

Let $\boldsymbol{I}=\left\{i_{1}, i_{2}, \ldots, i_{\mathrm{n}}\right\}$ be a set of all items, $\boldsymbol{D} \boldsymbol{B}$ be a transaction database. The external utility of item $\boldsymbol{i}_{\boldsymbol{k}} \in \boldsymbol{I}, \boldsymbol{s}\left(\boldsymbol{i}_{\boldsymbol{k}}\right)$, is the value associated with item $\boldsymbol{i}_{\boldsymbol{k}}$ in the utility table. The external utility reflects how useful an item is. For example, in Table 1, the external utility of item $\mathrm{A}, \mathrm{s}(\mathrm{A})$, is 5 .

Table 1. An example of utility table and transaction table

\begin{tabular}{|c|c|}
\hline Item & Profit $(\$$ \\
\hline A & 5 \\
\hline B & 1 \\
\hline C & 13 \\
\hline D & 50 \\
\hline
\end{tabular}

\begin{tabular}{|c|c|c|c|c|}
\hline $\mathrm{TID}$ & $\mathrm{A}$ & $\mathrm{B}$ & $\mathrm{C}$ & $\mathrm{D}$ \\
\hline $\mathrm{T}_{1}$ & 0 & 0 & 3 & 5 \\
\hline $\mathrm{T}_{2}$ & 1 & 2 & 0 & 0 \\
\hline $\mathrm{T}_{3}$ & 0 & 3 & 1 & 0 \\
\hline $\mathrm{T}_{4}$ & 5 & 0 & 1 & 0 \\
\hline $\mathrm{T}_{5}$ & 1 & 0 & 0 & 2 \\
\hline $\mathrm{T}_{6}$ & 0 & 0 & 1 & 2 \\
\hline $\mathrm{T}_{7}$ & 3 & 1 & 0 & 1 \\
\hline $\mathrm{T}_{8}$ & 1 & 0 & 3 & 3 \\
\hline $\mathrm{T}_{9}$ & 0 & 5 & 0 & 0 \\
\hline $\mathrm{T}_{10}$ & 0 & 1 & 1 & 0 \\
\hline
\end{tabular}

The utility of item $\boldsymbol{i}_{\boldsymbol{k}} \in \boldsymbol{I}$ in transaction $\boldsymbol{T}_{\boldsymbol{p}}, \boldsymbol{u}\left(\boldsymbol{i}_{\boldsymbol{k}}, \boldsymbol{T}_{\boldsymbol{p}}\right)$, is the quantitative measure of utility for item $\boldsymbol{i}_{\boldsymbol{k}}$ in transaction $\boldsymbol{T}_{\boldsymbol{p}}$. For 
example, in Table $2, \mathrm{u}\left(\mathrm{A}, \boldsymbol{T}_{4}\right)=5 \times 5=25$. Let $\mathrm{u}\left(\boldsymbol{X}, \boldsymbol{T}_{\boldsymbol{p}}\right)$ be the utility of itemset $\boldsymbol{X}$ in transaction $\boldsymbol{T}_{\boldsymbol{p}}$. For example, $\mathrm{u}\left(\{\mathrm{ABD}\}, \mathrm{T}_{7}\right)$ $=3 \times 5+1 \times 1+1 \times 50=66$. Let $\mathrm{u}(\boldsymbol{X})$ be the utility of itemset $\boldsymbol{X}$, which is the sum of the utilities of $\boldsymbol{X}$ in all transaction containing $\boldsymbol{X}$. For example, $\boldsymbol{X}=\{\mathrm{A}, \mathrm{B}\}, \mathrm{u}(\boldsymbol{X})=\mathrm{u}\left(\boldsymbol{X}, \boldsymbol{T}_{2}\right)+\mathrm{u}\left(\boldsymbol{X}, \boldsymbol{T}_{7}\right)=25+16$ $=41$.

Let $\boldsymbol{u}$ be the minimum threshold, the utility mining is to find all itemset with utility will be greater than or equal to $\boldsymbol{u}$. However, the downward closure property does not hold for the utility mining. That is, a high utility itemset may contain some low utility itemsets. In Table 1, we assume that the minimum utility threshold is 100 . The itemset $\{\mathrm{ACD}\}$ is a high utility itemset, because $u(\{A C D\})=194$. The itemset $\{A C\}$ is a sub-itemset of $\{A C D\}$, and $u(\{A C\})=82$. Therefore, it is a difficult work to find efficiently all high utility itemsets, and it's also a challenge to restrict the size of the candidate set.

Although the downward closure property does not hold in the utility model. Liu et al. [16], [17] proposed the Two-Phase algorithm to set up the transaction-weight utility which can comply with the transaction-weight downward closure property. The definition as follows: The transaction utility of transaction $\boldsymbol{T}_{\boldsymbol{i}}$, denoted as $\operatorname{tu}\left(\boldsymbol{T}_{\boldsymbol{i}}\right)$, is the sum of utilities of all items in $\boldsymbol{T}_{\boldsymbol{i}}$. The transaction-weighted utilization of an itemset $\boldsymbol{X}$, denoted as $\operatorname{twu}(\boldsymbol{X})$, is the sum of the transaction utilities of all the transactions containing $\boldsymbol{X}$.

Table 2. Transaction utility of the transaction database

\begin{tabular}{|c|c|c|c|}
\hline TID & Transaction & TID & Transaction \\
\hline $\mathrm{T}_{1}$ & 289 & $\mathrm{~T}_{6}$ & 113 \\
\hline $\mathrm{T}_{2}$ & 25 & $\mathrm{~T}_{7}$ & 66 \\
\hline $\mathrm{T}_{3}$ & 16 & $\mathrm{~T}_{8}$ & 194 \\
\hline $\mathrm{T}_{4}$ & 38 & $\mathrm{~T}_{9}$ & 5 \\
\hline $\mathrm{T}_{5}$ & 105 & $\mathrm{~T}_{10}$ & 28 \\
\hline
\end{tabular}

For example, in Table 2, twu $(\mathrm{B})=\mathrm{tu}\left(\mathrm{T}_{2}\right)+\mathrm{tu}\left(\mathrm{T}_{3}\right)+\mathrm{tu}\left(\mathrm{T}_{7}\right)+\mathrm{tu}\left(\mathrm{T}_{9}\right)$ $+\operatorname{tu}\left(\mathrm{T}_{10}\right)=140, \operatorname{twu}(\mathrm{C})=\operatorname{tu}\left(\mathrm{T}_{1}\right)+\mathrm{tu}\left(\mathrm{T}_{3}\right)+\mathrm{tu}\left(\mathrm{T}_{4}\right)+\mathrm{tu}\left(\mathrm{T}_{6}\right)+\mathrm{tu}\left(\mathrm{T}_{8}\right)$ $+\operatorname{tu}\left(\mathrm{T}_{10}\right)=678$ and $\operatorname{twu}(\{\mathrm{BC}\})=\operatorname{tu}\left(\mathrm{T}_{3}\right)+\operatorname{tu}\left(\mathrm{T}_{10}\right)=44$. If the minimum threshold is 40 , itemset $\{\mathrm{BC}\}$ becomes a high transaction-weight utilization itemset. Its sub-itemset $\{B\}$ and $\{C\}$ are also high transaction-weight utilization itemsets.

In addition, Li et al. [18] proposed the share framework to develop FSM-algorithm which takes advantage of the level closure property to discover all high share itemsets. And then Li et al. [19] proposed an Enhanced FSM-algorithm to improve the performance of FSM-algorithm. Li et al. also proposed the ShFSM-algorithm to efficiently lower the number of useless candidates.

\subsection{Temporal Data Mining}

Temporal data mining searches for interesting correlations or patterns in large sets of temporal data. Chang et al. [18] introduced research for temporal association rule mining. Traditional association rule algorithms can't find the temporal association rules in the particular periods.

Temporal association rules mining doesn't consider the utility of every item. Temporal utility mining is a research which is extended from temporal association rules mining and utility mining. Tseng et al. [20] introduced an efficient algorithm, THUIMine, to finding temporal high utility itemsets from data streams. THUI-Mine was based on the principle of Two-Phase algorithm
[21], and was extended by SWF-algorithm to be applicable in incremental database. The method can effective reduce the number of 2-itemset candidates, and used the same way to generate $\mathrm{k}$-itemset $(\mathrm{k} \geq 2)$ candidates.

\section{PROPOSED ALGORITHMS}

Let $\left\{i_{1}, i_{2}, \ldots, i_{\mathrm{m}}\right\}$ be a set of all items, $\boldsymbol{D} \boldsymbol{B}$ be the original transaction database segmented into $n$ partitions $\left\{P_{1}, P_{2}, \ldots, P_{n}\right\}$. Let $\mathrm{u}(\mathrm{X})$ bs the utility of itemset $\boldsymbol{X}$. The transaction-weighted utilization of an itemset $\boldsymbol{X}$, denoted as $\operatorname{twu}(\boldsymbol{X})$, is the sum of the transaction utilities of all the transactions containing $X$. The high temporal utility itemsets are the itemsets with utilities greater than or equal to a specified threshold in particular periods. That is, the high temporal utility itemsets are the high utility itemsets for particular periods. The incremental utility mining is to find all high temporal utility itemsets. The definitions of other notations used in this paper are given in Table 3.

Table 3. Definitions of notations

\begin{tabular}{|c|l|}
\hline$d b^{[\mathrm{i}, \mathrm{j}]}$ & Partition database from $P_{i}$ to $P_{j}$, where $\mathrm{i}<\mathrm{j}$. \\
\hline$s$ & Utility threshold in each partition. \\
\hline$L U^{[\mathrm{i}, \mathrm{j}]}$ & High temporal utility itemsets in $P_{i}$ to $P_{j}$. \\
\hline$R U^{k}$ & High transaction-weight utility itemsets in partition $k$. \\
\hline$C_{p}^{k}$ & The candidate $p$-itemsets in partition $k$. \\
\hline$I . u$ & The utility of itemset $I$. \\
\hline I.twu & The transaction utility of itemset $I$. \\
\hline pcoun & The number of partitions. \\
\hline I.start & The starting partition when $I$ was added to $C_{p}^{k}$. \\
\hline$d b^{+}$ & The added portion of an on-going transaction database. \\
\hline
\end{tabular}

\subsection{IUM and FIUM Algorithms}

In this section, the paper proposes two efficient algorithms, Incremental Utility Mining (IUM) Algorithm and Fast Incremental Utility Mining (FIUM) Algorithm, for incremental utility mining. IUM-Algorithm is based on the Two-Phase algorithm [17], [18] and FIUM-Algorithm is based on the ShFSM-algorithm [19]. The detail of IUM-Algorithm is shown in Figure 1.

In Figure 1, the IUM-Algorithm first finds the 1-itemset from the first partition $\mathrm{P}_{1}$ of the original transaction database, and then it determines whether the utilities of the 1-itemsets or the transaction-weight utilities of the 1-itemsets are greater than or equal to the minimum utility threshold. If the transaction-weight utilities of the 1-itemsets are greater than or equal to the minimum utility threshold, those itemsets are saved in $R U^{I}{ }_{1}$. If the utilities of the 1-itemsets are greater than or equal to the minimum utility threshold, those itemsets are saved in $L U^{[1,1]}{ }_{1}$. In fact, the transaction-weight utility of an itemset is always greater than or equal to its utility. Moreover we have that $L U^{[1,1]} \subseteq R U_{1}^{l}$. The 1itemsets in $R U^{l}{ }_{1}$ will be used to generate the level 2 candidates, denoted as $C_{2}^{l}$, which are based on Candidate-gen Algorithm. Like the previous steps, the 2-itemsets in $C^{l}{ }_{2}$ with high utilities and high transaction-weight utilities will be saved in $L U^{[l, I]}{ }_{2}$ and $R U^{l}{ }_{2}$, respectively. The process continues until no candidate is generated in $P_{1} . L U^{[1,1]}$ will be saved and evaluated in the phase of $P_{2}$. 


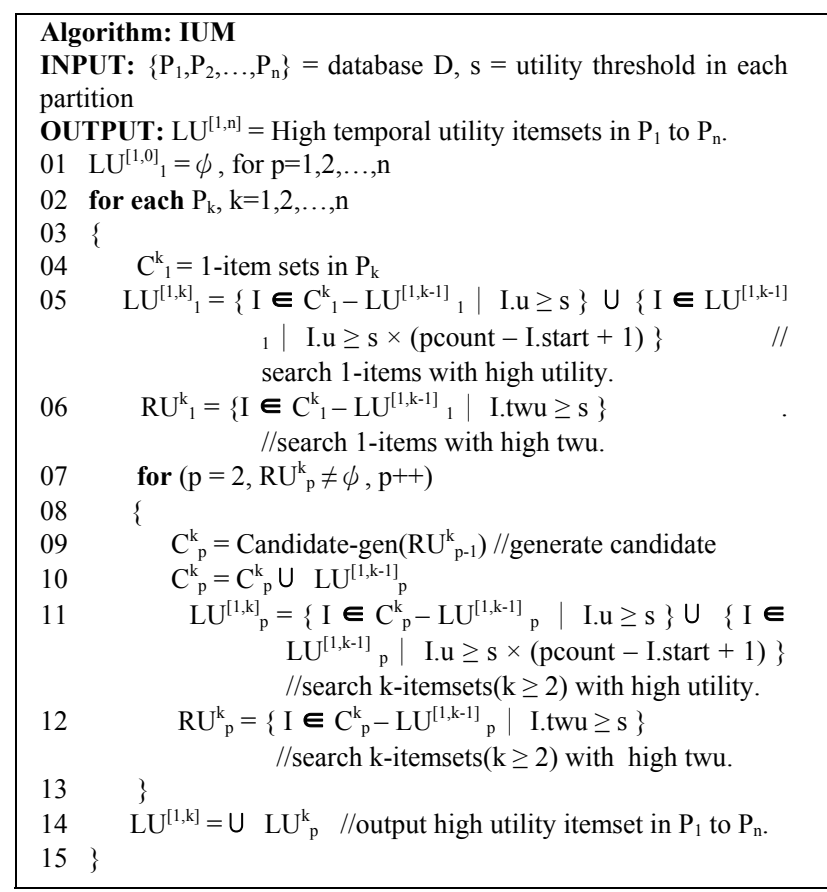

Figure 1. The procedure of IUM-Algorithm

In the temporal database $d b^{[1,2]}$, which is the union of the partitions $P_{1}$ and $P_{2}$, the threshold for itemsets carried out from the previous phase (that is, the itemsets in $L U^{[1,1]}$ ) is threshold $\times 2$ and for newly identified itemsets is original threshold. In the same way, the algorithm will find the1-itemset from the partition $P_{2}$, and then it determines whether the utilities of the 1-itemsets or the transaction-weight utilities of the 1-itemsets are greater than or equal to the minimum utility threshold. If the transaction-weight utilities of the 1-itemsets are greater than or equal to the minimum utility threshold, those itemsets are saved in $R U^{2}{ }_{1}$. If the utilities of the 1-itemsets are greater than or equal to the minimum utility threshold, those itemsets are saved in $L U^{[1,2]}{ }_{1}$. Consequently, the algorithm will find the results of $L U^{[1,2]}$ and $R U^{2}$.

Repeating the process for all partitions, finally, the algorithm will output the result of $L U^{[1, n]}$ which is the set of all high temporal utility itemsets in $d b^{[1, n]}$. If the value of I.start of itemset $I$ in $L U^{[1, n]}$ is $\mathrm{k}$, we also have that $I$ is a high utility itemset for $d b^{[k, n]}$ which is the partition database from $P_{k}$ to $P_{n}$. If we want to know which itemsets are also high utility in the original transaction database $\boldsymbol{D}$, we just need to calculate the utility values of itemsets in $L U^{[1, n]}$. That is, the itemsets in $L U^{[l, n]}$ are the candidates of high utility itemsets in $\boldsymbol{D}$.

The procedure of FIUM-Algorithm is similar to IUM-Algorithm. The difference between two algorithms is the generation of candidates. FIUM-Algorithm also used the transaction-weight downward closure property to prune the candidates. In the step of the candidate generation for p-itemsets, IUM-Algorithm joins two candidates of $R U_{\mathrm{p}-1}^{k}$, but FIUM-Algorithm joins the itemsets of $R U^{k}{ }_{1}$ and $R U_{\mathrm{p}-1}^{k}$. For example, consider (p-1)-itemset candidate $X_{I}$ $\in R U_{\mathrm{p}-1}^{k}$, said $X_{1}=\left\{i_{1}, i_{2}, \ldots, i_{\mathrm{p}-1}\right\}$, and $X_{2} \in \mathrm{RU}^{k}{ }_{1}$, said $X_{2}=\left\{i_{q}\right\}$. If $i_{q}>i_{p-1}$, then $X=\left\{i_{1}, i_{2}, \ldots, i_{\mathrm{p}-1}, i_{\mathrm{q}}\right\}$ is a p-itemset candidate. In IUM-Algorithm, the time complexity of the $C_{p}^{k}$ generation is

$\mathrm{O}\left(\mathrm{n}^{2 \mathrm{p}-2}\right)$, where $\mathrm{n}$ is the number of $R U_{p-1}^{k}$. However, the time complexity of FIUM-Algorithm generation is diminished to $\mathrm{O}\left(\mathrm{n}^{\mathrm{p}}\right)$.

\subsection{An Example for Incremental Utility Mining Algorithm}

To illustrate how IUM-Algorithm works, we use the sample database in Table 4. In Table 4 (A), the database is first divided into three partitions $\left\{P_{1}, P_{2}, P_{3}\right\}$. Every partition has three transactions. The utility of each item is listed in Table 4 (B). Table 4 (C) lists the transaction-weight utility of each transaction.

Table 4. The sample database for IUM-Algorithm

(A)

\begin{tabular}{|c|c|c|c|c|c|c|}
\hline & & $\mathrm{A}$ & $\mathrm{B}$ & $\mathrm{C}$ & $\mathrm{D}$ & $\mathrm{E}$ \\
\hline \multirow{3}{*}{$\mathrm{P}_{1}$} & $\mathrm{~T}_{1}$ & 2 & 0 & 3 & 1 & 1 \\
\cline { 2 - 7 } & $\mathrm{T}_{2}$ & 0 & 1 & 5 & 0 & 2 \\
\cline { 2 - 7 } & $\mathrm{T}_{3}$ & 3 & 2 & 0 & 1 & 0 \\
\hline \multirow{3}{*}{$\mathrm{P}_{2}$} & $\mathrm{~T}_{4}$ & 0 & 2 & 1 & 8 & 0 \\
\cline { 2 - 7 } & $\mathrm{T}_{5}$ & 1 & 0 & 0 & 2 & 0 \\
\cline { 2 - 7 } & $\mathrm{T}_{6}$ & 2 & 3 & 0 & 7 & 4 \\
\hline \multirow{3}{*}{$\mathrm{P}_{3}$} & $\mathrm{~T}_{7}$ & 0 & 0 & 1 & 1 & 0 \\
\cline { 2 - 7 } & $\mathrm{T}_{8}$ & 0 & 2 & 4 & 5 & 3 \\
\cline { 2 - 7 } & $\mathrm{T}_{9}$ & 5 & 2 & 1 & 0 & 1 \\
\hline
\end{tabular}

(C)

\begin{tabular}{|c|c|c|c|}
\hline TID & Transaction & TID & Transaction \\
\hline $\mathrm{T}_{1}$ & 62 & $\mathrm{~T}_{7}$ & 25 \\
\hline $\mathrm{T}_{2}$ & 100 & $\mathrm{~T}_{8}$ & 105 \\
\hline $\mathrm{T}_{3}$ & 34 & $\mathrm{~T}_{9}$ & 50 \\
\hline $\mathrm{T}_{4}$ & 33 & $\mathrm{~T}_{10}$ & 16 \\
\hline $\mathrm{T}_{5}$ & 23 & $\mathrm{~T}_{11}$ & 62 \\
\hline $\mathrm{T}_{6}$ & 68 & $\mathrm{~T}_{12}$ & 23 \\
\hline
\end{tabular}

Table 5. High temporal utility itemsets generated from database by IUM-Algorithm
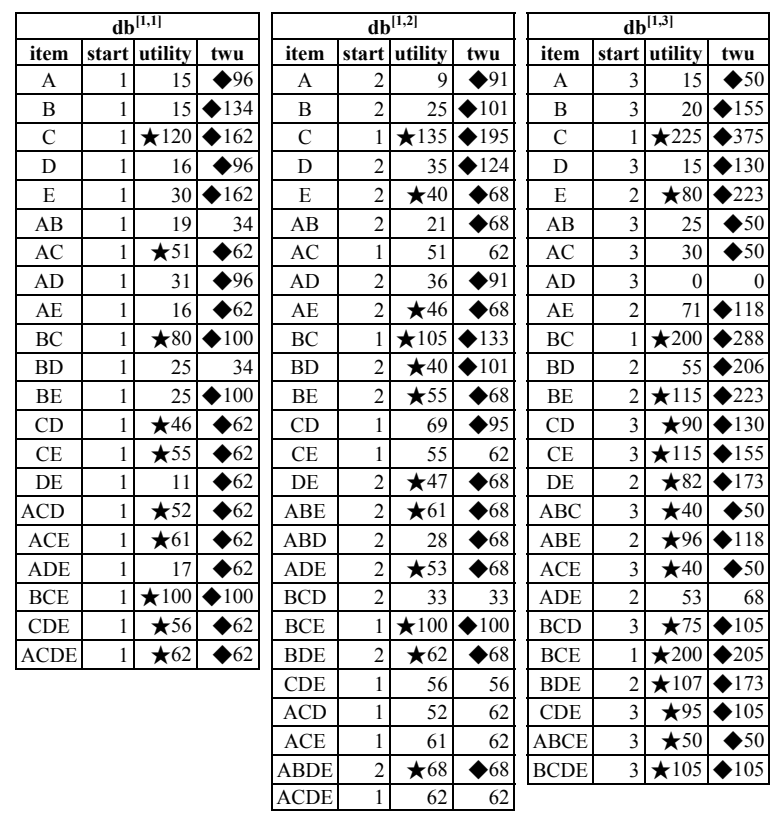

Table 5 lists the high temporal utility itemsets and high transaction-weight utility itemsets of the temporal database. In Partition $P_{l}, 1$-itemsets $\{$ A, B, C, D, E\} are found in Table 4(A). The utility value and transaction-weight utility (twu) are 
calculated for each candidate itemset. We assume that minimum utility threshold is 120 . Since there are three partitions, the utility threshold for $\mathrm{P}_{1}$ is $120 / 3=40$. In $P_{I}$ (or denoted as $d b^{[1, I]}$ ), I.start $=1$ for each itemset. Itemsets with utility or twu greater then 40 are selected. We can find $L U^{[1,1]}{ }_{I}=\{\mathrm{C}\}$ marked by " $\star$ ", $R U^{l}{ }_{I}=$ $\{\mathrm{A}, \mathrm{B}, \mathrm{C}, \mathrm{D}, \mathrm{E}\}=C^{l}{ }_{1}$ marked by " $\diamond$ ". $C^{l}{ }_{2}$ can be generated from the items in $R U^{l}{ }_{1}$, so $C^{l}{ }_{2}=\{\mathrm{AB}, \mathrm{AC}, \mathrm{AD}, \mathrm{AE}, \mathrm{BC}, \mathrm{BD}, \mathrm{BE}, \mathrm{CD}, \mathrm{CE}$, $\mathrm{DE}\}$. Similarly, we can find $L U^{[l, 1]}=\{\mathrm{AC}, \mathrm{BC}, \mathrm{CD}, \mathrm{CE}\}, R U^{l}{ }_{2}=$ $\{\mathrm{AC}, \mathrm{AD}, \mathrm{AE}, \mathrm{BC}, \mathrm{BE}, \mathrm{CD}, \mathrm{CE}, \mathrm{DE}\} . C^{l}{ }_{3}$ can be generated from the items in $R U^{l}{ }_{2}$, for example, itemset $\{\mathrm{ACD}\}$ is joined by $\{\mathrm{AC}\}$ and $\{A D\}$, and itemset $\{B C E\}$ is joined by $\{B C\}$ and $\{B E\}$. So $C_{3}^{l}=\{\mathrm{ACD}, \mathrm{ACE}, \mathrm{ADE}, \mathrm{BCE}, \mathrm{CDE}\}$. By calculating utility and twu for the itemsets in $C_{3}^{l}$, We have $L U^{[l, 1]}=\{\mathrm{ACD}, \mathrm{ACE}, \mathrm{BCE}$, $\mathrm{CDE}\}, R U^{l}{ }_{3}=\{\mathrm{ACD}, \mathrm{ACE}, \mathrm{BCE}, \mathrm{CDE}\}$. Consequently, we get $C_{4}^{l}=\{\mathrm{ACDE}\}, L U^{[l, l]}=\{\mathrm{ACDE}\}, R U_{3}^{l}=\{\mathrm{ACDE}\}$. In the phase of $\mathrm{P}_{1}, L U^{[l, l]}=\{\mathrm{C}, \mathrm{AC}, \mathrm{BC}, \mathrm{CD}, \mathrm{CE}, \mathrm{ACD}, \mathrm{ACE}, \mathrm{BCE}, \mathrm{CDE}$, ACDE $\}$ will be saved and evaluated in the phase of $P_{2}$.

In the temporal database $d b^{[1,2]}$, which is the union of the partitions $P_{1}$ and $P_{2}$, the threshold for itemsets carried out from the previous phase is $40+40=80$ and for newly identified itemsets is 40 . For example, $L U^{[1,1]}=\{\mathrm{C}, \mathrm{AC}, \mathrm{BC}, \mathrm{CD}, \mathrm{CE}, \mathrm{ACD}$, $\mathrm{ACE}, \mathrm{BCE}, \mathrm{CDE}, \mathrm{ACDE}\}$ is held in $d b^{[1,2]}$, and the threshold for itemsets in $L U^{[1,1]}$ is 80 . Item C has C.start $=1$, utility $=120+15=$ 135 , and twu $=162+33=195$. Both utility and twu of $\mathrm{C}$ are greater than 80 . Therefore, $\mathrm{C}$ is a high temporal utility itemset and a high transaction-weight utility itemset in $d b^{[1,2]}$.

On the other hand, we find that item A has A.start $=2$, utility $=9$, and twu $=91$. Since item A does not occur in $L U^{[1,1]}$ and the twu of $\mathrm{A}$ is greater than the threshold $40, \mathrm{~A}$ is a high transactionweight utility itemset in $d b^{[1,2]}$. We can find $L U^{[1,2]}{ }_{1}=\{\mathrm{C}, \mathrm{E}\}$, $R U^{2}{ }_{1}=\{\mathrm{A}, \mathrm{B}, \mathrm{C}, \mathrm{D}, \mathrm{E}\} . C^{2}{ }_{2}$ can be generated from the items in $R U^{2}{ }_{1}$, so $C_{2}^{2}=\{\mathrm{AB}, \mathrm{AC}, \mathrm{AD}, \mathrm{AE}, \mathrm{BC}, \mathrm{BD}, \mathrm{BE}, \mathrm{CD}, \mathrm{CE}, \mathrm{DE}\}$. Consequently, we can find $L U^{[1,2]}{ }_{2}=\{\mathrm{AE}, \mathrm{BC}, \mathrm{BD}, \mathrm{BE}, \mathrm{DE}\}$, $R U^{2}{ }_{2}=\{\mathrm{AB}, \mathrm{AE}, \mathrm{BC}, \mathrm{BD}, \mathrm{BE}, \mathrm{CD}, \mathrm{DE}\}, L U^{[1,2]}{ }_{3}=\{\mathrm{ABE}, \mathrm{ADE}$, $\mathrm{BCE}, \mathrm{BDE}\}, R U^{2}{ }_{2}=\{\mathrm{ABE}, \mathrm{ABD}, \mathrm{ADE}, \mathrm{BCE}, \mathrm{BDE}\}, L U^{[1,2]}{ }_{4}=$ $\{\mathrm{ABDE}\}$, and $R U^{2}{ }_{4}=\{\mathrm{ABDE}\}$. Therefore, $L U^{[1,3]}=\{\mathrm{C}, \mathrm{E}, \mathrm{AE}$, $\mathrm{BC}, \mathrm{BD}, \mathrm{BE}, \mathrm{DE}, \mathrm{ABE}, \mathrm{ADE}, \mathrm{BCE}, \mathrm{BDE}, \mathrm{ABDE}\}$.

In the temporal database $d b^{[1,3]}$, which is the union of $d b^{[1,2]}$ and partition $P_{3}$, the thresholds for itemsets carried out from partition $P_{1}$ and $P_{2}$ are $40+40+40=120$ and $40+40=80$, respectively. The threshold for newly identified itemsets is 40 . In conclusion, we can discover the final result $L U^{[1,3]}=\{\mathrm{C}, \mathrm{E}, \mathrm{BC}, \mathrm{BE}, \mathrm{CD}, \mathrm{CE}, \mathrm{DE}$, $\mathrm{ABC}, \mathrm{ABE}, \mathrm{ACE}, \mathrm{BCD}, \mathrm{BCE}, \mathrm{BDE}, \mathrm{CDE}, \mathrm{ABCE}, \mathrm{BCDE}\}$.

\section{EXPERIMENTAL STUDIES}

\subsection{Experimental environment}

All experiments were performed on a Pentium IV $3.40 \mathrm{GHz} \mathrm{CPU}$ with 2 GB RAM and Microsoft Windows XP PC. IUMAlgorithm and FIUM-Algorithm were coded in Microsoft Visual $\mathrm{C}++6.0$ and the experiment datasets were generated by IBM Data Generator [20]. The parameters of datasets were shown in Table 6.

Table 6. Parameters of datasets

\begin{tabular}{|c|c|c|c|}
\hline Dataset & $\begin{array}{c}\text { Number of } \\
\text { transactions } \\
\text { (ntrans) }\end{array}$ & $\begin{array}{c}\text { Average } \\
\text { transaction } \\
\text { length (slen) }\end{array}$ & $\begin{array}{c}\text { Number of } \\
\text { items } \\
\text { (nitems) }\end{array}$ \\
\hline $\begin{array}{c}\text { data.ntrans_10.nitems_0.0 } \\
\text { 2 }\end{array}$ & 10000 & 10 & 20 \\
\hline data.ntrans_10.nitems_0.1 & 10000 & 10 & 100 \\
\hline
\end{tabular}

\subsection{Performance on IUM-Algorithm and FIUM-Algorithm}

In this section, the experiments first performed on the two datasets by IUM-Algorithm and FIUM-Algorithm. Tables 7 shows the generation of candidates for two algorithms in two datasets with different utility threshold $\boldsymbol{u}$, where $\boldsymbol{u}=\alpha \times$ the total utility value of Partition 1 . The number of candidates of IUMAlgorithm and FIUM-Algorithm are the same in every partition. The right 3 columns of Table 7(A) and Table 7(B) list the numbers of the temporal high utility itemsets which start in each partition. Some temporal high utility itemsets generated from Partition 1 and some generated from Partition 2 and Partition 3. Because of the utilities of candidates which were generated from Partition 1 can not accumulate enough values to rise to transcend the minimum partition threshold, those candidates will be pruned in the first partition. Some new high temporal itemsets were generated from Partition 2 and Partition 3, because the utilities of those itemsets were greater than or equal to the minimum partition thresholds in Partition 2 and Partition 3. Another reason is that there are some new items which never occur in Partition 1, but the utilities of these new itemsets were greater than or equal to the minimum partition threshold.

Table 7. The number of candidate itemsets generated by IUMAlgorithm and FIUM-Algorithm

\begin{tabular}{|c|c|c|c|c|c|c|c|c|c|c|c|c|c|}
\hline \multicolumn{7}{|c|}{ (A) } & \multicolumn{7}{|c|}{ (B) } \\
\hline \multicolumn{7}{|c|}{ data.ntrans_10.nitems_0.02 $(\alpha=8 \%)$} & \multicolumn{7}{|c|}{ data.ntrans_10.nitems_0.1 $(\alpha=0.6 \%)$} \\
\hline $\begin{array}{l}\text { Candidate } \\
\text { Cand }\end{array}$ & IUM & FIUM & $\mathbf{L U}$ & \multicolumn{3}{|c|}{ Start } & \begin{tabular}{|l|} 
Candidate \\
\end{tabular} & IUM & FIUM & $\mathbf{L U}$ & \multicolumn{3}{|c|}{ start } \\
\hline \multicolumn{4}{|c|}{$\mathrm{db}^{[1,1]}$} & 1 & 2 & 3 & \multicolumn{4}{|c|}{$\mathrm{db}^{[1,1]}$} & 1 & 2 & 3 \\
\hline $\mathrm{C}_{2}$ & 171 & 171 & 20 & 20 & 0 & 0 & $\mathrm{C}_{2}$ & 4371 & 4371 & 395 & 395 & 0 & 0 \\
\hline $\mathrm{C}_{3}$ & 810 & 810 & 82 & 82 & 0 & 0 & $\mathrm{C}_{3}$ & 47568 & 47568 & 235 & 235 & 0 & 0 \\
\hline $\mathrm{C}_{4}$ & 2033 & 2033 & 143 & 143 & 0 & 0 & $\mathrm{C}_{4}$ & 29476 & 29476 & 12 & 12 & 0 & 0 \\
\hline $\mathrm{C}_{5}$ & 2281 & 2281 & 114 & 114 & 0 & 0 & $\mathrm{C}_{5}$ & 2104 & 2104 & 0 & 0 & 0 & 0 \\
\hline $\mathrm{C}_{6}$ & 1385 & 1385 & 44 & 44 & 0 & 0 & $\mathrm{C}_{6}$ & 51 & 51 & 0 & 0 & 0 & 0 \\
\hline $\mathrm{C}_{7}$ & 362 & 362 & 8 & 8 & 0 & 0 & $\mathrm{C}_{7}$ & 0 & 0 & 0 & 0 & 0 & 0 \\
\hline $\mathrm{C}_{8}$ & 39 & 39 & 0 & 0 & 0 & 0 & \multicolumn{4}{|c|}{$\mathbf{d b}^{[1,2]}$} & 1 & 2 & 3 \\
\hline $\mathrm{C}_{9}$ & 0 & 0 & 0 & 0 & 0 & 0 & $\mathrm{C}_{2}$ & \begin{tabular}{|l|l|}
4753 \\
\end{tabular} & 4753 & 445 & 395 & 50 & 0 \\
\hline \multicolumn{4}{|c|}{$\mathrm{db}^{[1,2]}$} & 1 & 2 & 3 & $\mathrm{C}_{3}$ & 88926 & 88926 & 300 & 235 & 65 & 0 \\
\hline $\mathrm{C}_{2}$ & 190 & 190 & 23 & 20 & 3 & 0 & $\mathrm{C}_{4}$ & 234453 & 234453 & 19 & 12 & 7 & 0 \\
\hline $\mathrm{C}_{3}$ & 936 & 936 & 99 & 82 & 17 & 0 & $\mathrm{C}_{5}$ & 68654 & 68654 & 0 & 0 & 0 & 0 \\
\hline $\mathrm{C}_{4}$ & 3240 & 3240 & 167 & 143 & 24 & 0 & $\mathrm{C}_{6}$ & 4020 & 4020 & 0 & 0 & 0 & 0 \\
\hline $\mathrm{C}_{5}$ & 8254 & 8254 & 133 & 114 & 19 & 0 & $\mathrm{C}_{7}$ & 214 & 214 & 0 & 0 & 0 & 0 \\
\hline $\mathrm{C}_{6}$ & 13430 & 13430 & 51 & 44 & 7 & 0 & $\mathrm{C}_{8}$ & 31 & 31 & 0 & 0 & 0 & 0 \\
\hline $\mathrm{C}_{7}$ & 13887 & 13887 & 9 & 8 & 1 & 0 & $\mathrm{C}_{9}$ & 3 & 3 & 0 & 0 & 0 & 0 \\
\hline $\mathrm{C}_{8}$ & 9029 & 9029 & 0 & 0 & 0 & 0 & $\mathrm{C}_{10}$ & 0 & 0 & 0 & 0 & 0 & 0 \\
\hline $\mathrm{C}_{9}$ & 3319 & 3319 & 0 & 0 & 0 & 0 & \multicolumn{4}{|c|}{${d b^{[1,3]}}^{[1,3}$} & 1 & 2 & 3 \\
\hline $\mathrm{C}_{10}$ & 584 & 584 & 0 & 0 & 0 & 0 & $\mathrm{C}_{2}$ & \begin{tabular}{|l|}
4851 \\
\end{tabular} & 4851 & \begin{tabular}{|l|}
608 \\
\end{tabular} & 395 & 50 & 163 \\
\hline $\mathrm{C}_{11}$ & 33 & 33 & 0 & 0 & 0 & 0 & $\mathrm{C}_{3}$ & 108669 & 108669 & \begin{tabular}{|l|}
602 \\
\end{tabular} & 235 & 65 & 302 \\
\hline $\mathrm{C}_{12}$ & 0 & 0 & 0 & 0 & 0 & 0 & $\mathrm{C}_{4}$ & 571774 & 571774 & \begin{tabular}{|l|}
129 \\
\end{tabular} & 12 & 7 & 110 \\
\hline \multicolumn{4}{|c|}{$\mathrm{db}^{[1,3]}$} & 1 & 2 & 3 & $\mathrm{C}_{5}$ & 387832 & 387832 & \begin{tabular}{|l|}
0 \\
\end{tabular} & 0 & 0 & 0 \\
\hline $\mathrm{C}_{2}$ & 190 & 190 & 23 & 20 & 3 & 0 & $\mathrm{C}_{6}$ & 49057 & 49057 & 0 & 0 & 0 & 0 \\
\hline $\mathrm{C}_{3}$ & 984 & 984 & 212 & 82 & 17 & 113 & $\mathrm{C}_{7}$ & 2345 & 2345 & 0 & 0 & 0 & 0 \\
\hline $\mathrm{C}_{4}$ & 3466 & 3466 & 352 & 143 & 24 & 185 & $\mathrm{C}_{8}$ & 235 & 235 & 0 & 0 & 0 & 0 \\
\hline $\mathrm{C}_{5}$ & 8599 & 8599 & \begin{tabular}{|l|}
327 \\
\end{tabular} & 114 & 19 & 194 & $\mathrm{C}_{9}$ & 30 & 30 & 0 & 0 & 0 & 0 \\
\hline $\mathrm{C}_{6}$ & 14176 & 14176 & \begin{tabular}{|l|}
193 \\
\end{tabular} & 44 & 7 & 142 & $\mathrm{C}_{10}$ & 2 & 2 & 0 & 0 & 0 & 0 \\
\hline $\mathrm{C}_{7}$ & \begin{tabular}{|l|}
15831 \\
\end{tabular} & 15831 & \begin{tabular}{|l|}
57 \\
\end{tabular} & 8 & 1 & 48 & $\mathrm{C}_{11}$ & 0 & 0 & 0 & 0 & 0 & 0 \\
\hline $\mathrm{C}_{8}$ & 11461 & 11461 & 22 & 0 & 0 & 22 & & & & & & & \\
\hline $\mathrm{C}_{9}$ & \begin{tabular}{|l|}
5199 \\
\end{tabular} & \begin{tabular}{|l|l|}
5199 \\
\end{tabular} & 0 & 0 & 0 & 0 & & & & & & & \\
\hline $\mathrm{C}_{10}$ & 1281 & 1281 & 0 & 0 & 0 & 0 & & & & & & & \\
\hline $\mathrm{C}_{11}$ & 115 & 115 & 0 & 0 & 0 & 0 & & & & & & & \\
\hline $\mathrm{C}_{12}$ & 0 & 0 & 0 & 0 & 0 & 0 & & & & & & & \\
\hline
\end{tabular}



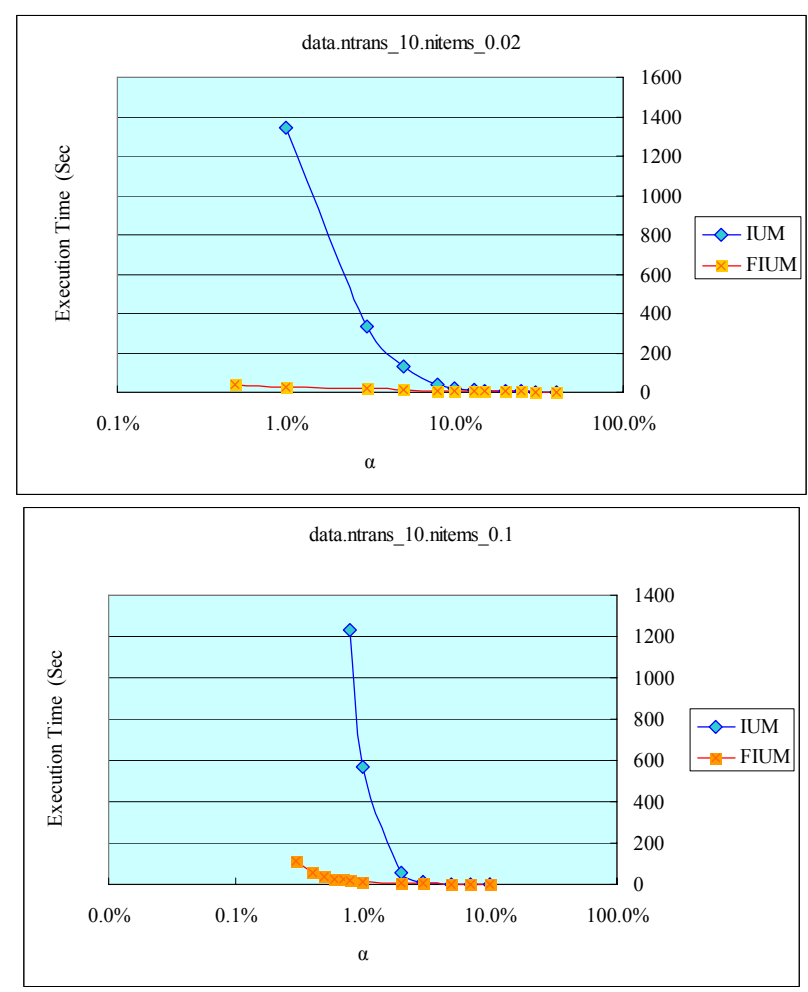

Figure 2. Execution time for IUM-Algorithm and FIUMAlgorithm with various minimum utility thresholds in two datasets

Figure 2 shows that the performance in the execution times of IUM-Algorithm and FIUM-Algorithm on two datasets with various minimum utility thresholds. The FIUM-Algorithm is more efficiently than IUM-Algorithm by the experimental result. Because of FIUM-Algorithm spends less time in joining candidate itemsets than IUM-Algorithm does.

\section{CONCLUSIONS}

The study proposed novel incremental utility mining methods, IUM-Algorithm, and FIUM-Algorithm, which can discover temporal high utility itemsets. The algorithms can efficiently identify all high temporal utility itemsets that users will be interested in particular periods when the new transaction data are added into the original transaction database. The algorithm not only can find the temporal high utility itemsets for particular time periods, but also can find the high utility itemsets for the entire transaction database. In fact, the high temporal utility itemsets are the candidates of high utility itemsets.

Incremental utility mining strives for finding high temporal utility itemsets that drive large portions of utility in a particular period. However, it does not indicate how often such itemsets appear in the period. In the future, the temporal utility-frequent mining can be more investigated.

\section{REFERENCES}

[1] Agrawal, R. and Srikant, R. 1994. Fast Algorithms for Mining Association Rules. In Proceedings of the 20th VLDB Conference (Santiago, Chile). 487-499.
[2] Park, J. S., Chen, M. S., and Yu, P. S. 1995. An Effective Hash-based Algorithm for Mining Association Rules. In Proceedings of ACM SIGMOD Conf. on Management of Data. 175-186.

[3] Savasere, A., Oiecinski, E., and Navathe, S. 1995. An Efficient Algorithm for Mining Association Rules in Large Databases. In Proceedings of the 20th Int. Conf. on Very Large Data Bases. 432-444.

[4] Agrawal, R. and Srikant, R. 1996. Parallel Mining of Association Rules. IEEE Transactions on Knowledge and Data Engineering. 8, 6, 962-969.

[5] Agrawal, R. and Shim, K. 1996. Developing TightlyCoupled Data Mining Applications on a Relational Database System. In Proceedings of the 2nd Int. Conf. on Knowledge Discovery in Databases and Data Mining (Portland, Oregon). 287-290.

[6] Agrawal, R., Srikant, R., and Vu, Q. 1997. Mining Association Rules with Item Constraints. In Proceedings of the 3rd Int. Conf. on Knowledge Discovery in Database and Data Mining (Newport Beach, California). 67-73.

[7] Cheung, D., Han, J., Ng V., and Wong, C. Y. 1996. Maintenance of Discovered Association Rules in Large Databases: An Incremental Updating Technique. In Proceedings of the 12th Int. Conf. on Data Engineering (ICDE96). 106-114.

[8] Cheung, D., Lee, S.D., and Kao, B. 1997. A General Incremental Technique for Updating Discovered Association Rules. In Proceedings of Int. Conf. on Database Systems for Advanced Applications. 185-194.

[9] Hong, T. P., Wang, C. Y., and Tao, Y. H. 2000. Incremental Data Mining Based on Two Support Thresholds. In Proceedings of the 4th Int. Conf. on Knowledge-Based Intelligent Engineering Systems and Allied Technologies. 436-439.

[10] Lee, C. H., Lin, C. R., and Chen, M. S. 2001. SlidingWindow Filtering: An Efficient Algorithm for Incremental Mining. In Proceedings of the 10th Int. Conf. on Information and Knowledge Management. 263-270.

[11] Chang, C. H., Lin, C. R., and Chen, M. S. 2001. On Mining General Temporal Association Rules in A Publication Database. In Proceedings of 2001 IEEE Int. Conf. on Data Mining. 337-344.

[12] Masseglia, F., Poncelet, P., and Teisseire, M. 2003. Incremental Mining of Sequential Patterns in Large Databases. Data \& Knowledge Engineering. 46. 97-121.

[13] Cheng, H., Yang, X., and Han, J. 2004. IncSpan: Incremental Mining of Sequential Patterns in Large Database. In Proceedings of the 10th ACM SIGKDD Int. Conf. on Knowledge Discovery and Data Mining. 527-532.

[14] Yao, H., Hamilton, H. J., and Butz, C. J. 2004. A Foundational Approach to Mining Itemset Utilities from Databases. In Proceedings of the 4th SIAM Int. Conf. on Data Mining. 428-486.

[15] Tseng, V. S., Chu, C. J., and Liang, T. 2006. Efficient Mining of Temporal High Utility Itemsets from Data 
Streams. In Proceedings of ACM KDD Workshop on UtilityBased Data Mining.

[16] Liu, Y., Liao W. K., and Choudhary, A. 2005. A Fast High Utility Itemsets Mining Algorithm. In Proceedings of the 11th ACM SIGKDD Workshop on Utility-Based Data Mining.

[17] Liu, Y., Liao, W. K., and Choudhary, A. 2005. A Two-Phase Algorithm for Fast Discovery of High Utility Itemsets. LECTURE NOTES IN COMPUTER SCIENCE 3518, Springer-Verlag, Berlin Heidelberg. 689-695.

[18] Li, Y. C., Yeh, J. S., and Chang, C. C. 2005. A Fast Algorithm for Mining Share-Frequent Itemsets. In
Proceedings of the 7th Asia Pacific Web Conference, Springer-Verlag, Germany. 417-428.

[19] Li, Y. C., Yeh, J. S., and Chang, C. C. 2005. Efficient Algorithm for Mining Share-Frequent Itemsets. In Proceedings of Fuzzy Logic, Soft Computing and Computational Intelligence (IFSA 2005), Springer Tsinghua, China. 534-539.

[20] IBM Almaden Research Center, http://www.almaden.ibm.com/cs/projects/iis/hdb/Projects/dat a_mining/mining.shtml 